\title{
Médiévales
}

Langues, Textes, Histoire

62 | printemps 2012

Hagiographie et réforme dans l'Occident latin

\section{Hagiographie et réformes monastiques dans le monde franc du VII siècle}

Hagiography and Monastic Reforms in the Seventh-Century Frankish World

\author{
Anne-Marie Helvétius
}

\section{OpenEdition}

Journals

Édition électronique

URL : https://journals.openedition.org/medievales/6627

DOI : 10.4000/medievales.6627

ISSN : $1777-5892$

Éditeur

Presses universitaires de Vincennes

Édition imprimée

Date de publication : 2 juin 2012

Pagination : $33-47$

ISBN : 978-2-84292-346-4

ISSN : 0751-2708

Référence électronique

Anne-Marie Helvétius, "Hagiographie et réformes monastiques dans le monde franc du vII eiècle », Médiévales [En ligne], 62 I printemps 2012, mis en ligne le 28 juin 2012, consulté le 22 avril 2022. URL http://journals.openedition.org/medievales/6627 ; DOI : https://doi.org/10.4000/medievales.6627 
Médiévales 62,printemps 2012, p. 33-48

\author{
Anne-Marie HELVÉTIUS
}

\title{
HAGIOGRAPHIE ET RÉFORMES MONASTIQUES DANS LE MONDE FRANC DU VII SIÈCLE
}

Dans la tradition monastique ancienne, les ascètes étaient invités à suivre les pas d'un père vivant qui leur communiquait son enseignement et leur dispensait ses conseils au jour le jour, conformément à la première épître de Pierre qui encourage les pasteurs à être eux-mêmes la forma de leur troupeau ${ }^{1}$. À l'origine de la notion de «réforme», ce terme de forma désigne la mise en forme, l'organisation d'un troupeau par le pasteur. Les sources hagiographiques anciennes s'y réfèrent fréquemment; la Vie d'Hilaire d'Arles, par exemple, explique comment ce père spirituel qui avait institué une congrégation ascétique en Arles l'imprégnait de son exemple et la formait par sa parole ${ }^{2}$. Dans ce même sens littéral, l'inauguration d'une nouvelle communauté monastique est parfois qualifiée d'informatio ${ }^{3}$.

Par conséquent, la notion de réforme monastique peut s'appliquer à toute tentative de modification de la vie commune des moines, que l'on présente le plus souvent comme un retour à une forma originelle. Dans le cas d'un établissement particulier, celle-ci peut correspondre à l'organisation - réelle ou supposée - instituée par le père fondateur de la communauté en question. Dans

1. I Pierre 5, 3, d'après la Vulgate: «forma estote gregi».

2. BHL 3882: Honorat de Marseille, La Vie d'Hilaire d'Arles, éd. P.-A. JacoB, Paris, 1995 , 10, p. 112. La même idée apparaît déjà dans la Vie d'Honorat (BHL 3975): Hilaire D'ArLes, Vie de saint Honorat, 19, 1-3, éd. M.-D. VALENTIN, Paris, 1977, p. 124-126 et, un peu plus tard, dans la Vie de Séverin (BHL 7655) : EugIPPE, Vie de saint Séverin, éd. P. RÉGERAT, Paris, 1991, p. 158 (avec une citation de I Pierre 5, 3).

3. Par exemple dans la Vita patrum Jurensium (BHL 7309-5073-2665): Vie des Pères du Jura, 179, éd. F. Martine, Paris, 1968, p. 432 (à propos de la refondation de Saint-Maurice d'Agaune en $515)$. 
une perspective plus large, les promoteurs d'une réforme peuvent également se réclamer de leur fidélité à la tradition monastique en général, à l'héritage des anciens pères ou à tel ou tel père considéré comme une autorité 4 . À une époque où la notion de «règle monastique» est encore floue ${ }^{5}$, l'un des moyens utilisés pour faire connaître cette forma primitive ou l'héritage spirituel auquel on se réfère est l'écriture hagiographique. De fait, en milieu monastique, la Vita du saint patron ou du père fondateur offrait souvent aux moines un florilège d'exemples à imiter, de vertus à acquérir et de vices à proscrire. Déjà dans la Vie du premier père des moines, Athanase d'Alexandrie affirmait dans son prologue: «Pour des moines, en effet, la vie d'Antoine suffit comme modèle d'ascèse ${ }^{6} . »$ Dans le haut Moyen Âge, un certain nombre de Vies de saints présentent la particularité de privilégier l'exposition des vertus spirituelles de leur héros, au détriment des détails biographiques ou de la promotion de son culte. La plupart de ces textes ont été rédigés dans un contexte de réforme monastique, que ce soit pour appuyer une transformation du mode de vie présentée comme un retour aux valeurs promues par le saint patron ou, au contraire, pour y résister au nom d'une fidélité à ce que l'on considère comme la tradition d'origine. Les réécritures successives des Vies permettaient d'actualiser l'enseignement présumé du père fondateur au gré des circonstances ${ }^{7}$.

À l'époque précarolingienne, tandis que cohabitent diverses formes de monachisme dans le monde franc, le rôle politique de ces communautés ne cesse de croître. Une série de récits hagiographiques produits à cette époque dans ces milieux témoignent du lien étroit qui s'établit entre réforme monastique et unification politique. Dans cette perspective, la réforme acquiert une dimension universelle: au-delà de la vie commune des moines, ce sont les valeurs chrétiennes

4. Sur le sens du terme «réforme» en milieu monastique, voir notamment R. KotTJE et H. Maurer éd., Monastische Reformen im 9. und 10. Jahrhundert, Sigmaringen, 1989; et J. SemmLer, «Le monachisme occidental du $\mathrm{vIII}^{\mathrm{e}}$ au $\mathrm{x}^{\mathrm{e}}$ siècle: formation et réformation », Revue Bénédictine, 103 (1993), p. 68-89.

5. Sur la notion de règle, voir en dernier lieu A.-M. HelvéTIus, «Normes et pratiques de la vie monastique en Gaule avant 1050 : présentation des sources écrites », dans O. DeLouis et M. MossakowsKa-Gaubert éd., La Vie quotidienne des moines en Orient et en Occident (IV $V^{e} X^{e}$ siècle), I. Les sources, Le Caire, 2012 (sous presse).

6. Athanase d'Alexandrie, Vie d'Antoine, prol., 3, éd. G. J. M. Bartelink, Paris, 1994, p. 127. Jérôme montre ainsi le moine Hilarion s'efforçant d'imiter Antoine d'après sa Vie: JÉRÔME, Trois Vies de moines (Paul, Malchus, Hilarion), § 2, 5, éd. E. M. Morales et P. Leclerc, Paris, 2007, p. 216. Voir dans le même sens JeAn CASSIEN, Institutions cénobitiques, préf., 3, éd. J.-C. GuY, Paris, 1965 , p. 24.

7. Sur le procédé de la réécriture hagiographique, voir surtout M. GoulLET, Écriture et réécriture hagiographiques. Essai sur les Vies de saints dans l'Occident latin médiéval (VII'-XIII' s.), Turnhout, 2005; et EAD. et M. Heinzelmann éd., La Réécriture hagiographique dans l'Occident médiéval. Transformations formelles et idéologiques, Ostfildern, 2003. 
et donc le comportement de tous les chrétiens que l'on cherche à corriger. Afin de mieux appréhender les enjeux de ces courants réformateurs, il convient dans un premier temps de dresser un état des recherches actuelles. Quelques exemples précis permettront ensuite de mesurer l'intérêt et la richesse des sources hagiographiques mérovingiennes pour cette problématique, dès lors qu'on les confronte aux informations fournies par les autres sources conservées. Cette enquête montrera finalement l'ampleur des recherches qui restent à mener pour éclairer quelque peu le fonctionnement de la société mérovingienne.

\section{L'héritage du $\mathrm{VI}^{\mathrm{e}}$ siècle}

L'histoire des réformes monastiques en Gaule débute en Provence dès les $\mathrm{V}^{\mathrm{e}}$ et $\mathrm{VI}^{\mathrm{e}}$ siècles, lorsque des penseurs s'efforcent de faire évoluer l'idéal ascétique vers un monachisme organisé sous une forme de plus en plus cénobitique. Dans cette perspective, les œuvres de Jean Cassien constituent une première étape, car elles visent déjà à uniformiser les courants monastiques occidentaux en proposant aux moines un mode de vie idéal présenté comme la synthèse de toutes les traditions orientales antérieures ${ }^{8}$. Après lui, d'autres penseurs proches des milieux lériniens, tels Hilaire d'Arles, Fauste de Riez, Ennode de Pavie ou Césaire d'Arles, contribuent à promouvoir l'idéal cénobitique dans les milieux monastiques de la Gaule du Sud par la rédaction de différents types de textes - traités d'ascèse, premières règles écrites, sermons ou Vies de saints. À partir de l'exemple de la Vie d'Antoine de Lérins, composée au début du vi siècle par Ennode de Pavie dans une perspective réformatrice, Stéphane Gioanni a bien montré comment ces anciens moines de Lérins devenus évêques inscrivent l'idéal monastique dans le prolongement de la tradition apostolique, le considèrent comme le modèle de perfection chrétienne par excellence et s'efforcent de le diffuser dans le monde ${ }^{9}$. En se fondant sur les vertus monastiques, il s'agit déjà de réformer l'organisation de la société chrétienne dans son ensemble.

Si d'autres formes d'expérience ascétique existent très tôt dans d'autres régions de la Gaule - par exemple à Tours, à Trèves, dans le Jura, à Auxerre ou à Saint-Maurice d'Agaune -, c'est à partir de Lérins et d'Arles que se diffusent $\mathrm{au} \mathrm{VI}^{\mathrm{e}}$ siècle les idéaux de cette réforme que l'on peut qualifier de «régulière» :

8. Voir O. Chadwick, John Cassian. A Study in Primitive Monasticism, Cambridge, 1950 (rééd. 1968), et la bibliographie mise à jour dans JEAN CASSIEN, Conférences I-VII, éd. E. Pichery, Paris, 1955 (rééd. 2008).

9. S. Gioanni, «Une figure suspecte de la sainteté lérinienne: saint Antoine d'après la Vita Antoni d'Ennode de Pavie», Recherches augustiniennes et patristiques, 35 (2007), p. 133-187; ID., «"Être véritablement moine": les représentations de l'identité ascétique dans la pastorale lérinienne ( $\mathrm{v}^{\mathrm{e}}-\mathrm{v} \mathrm{l}^{\mathrm{e}}$ siècles)», dans Y. Codou et M. Lauwers éd., Lérins, une île sainte de l'Antiquité au Moyen Âge, Turnhout, 2009, p. 141-165. 
il ne s'agit pas encore d'imposer l'observance rigoureuse aux préceptes d'une règle écrite unique, mais seulement d'inviter les moines et les moniales à entamer leur parcours de perfection au sein d'un établissement cénobitique, où les règles contraignantes de la vie en communauté les «formeront» à l'apprentissage des vertus chrétiennes par excellence que sont le renoncement aux biens matériels et aux plaisirs du siècle, l'obéissance, l'humilité, l'amour du prochain, la pénitence et la lutte permanente contre les tentations. Par extension, ces vertus sont recommandées à tous les chrétiens, qu'ils soient moines, clercs ou laïcs: si chacun s'efforçait de corriger ses mœurs à partir de ce modèle, la société toute entière deviendrait véritablement chrétienne.

Dès le $\mathrm{VI}^{\mathrm{e}}$ siècle, ces tentatives réformatrices suscitent des controverses. D'une part, certaines communautés s'efforcent de présenter leur mode de vie comme le plus parfait, générant ainsi un climat de concurrence au sein du monde monastique. D'autre part, ces vertus «régulières» sont jugées incompatibles avec les obligations de la cura animarum, qui contraignent les membres du clergé à vivre dans le siècle: comment concilier la vie active avec la vie contemplative ? Les clercs sont-ils «moins parfaits» que les moines? Quelles doivent être les relations entre évêques, clercs et moines? Un autre modèle d'hagiographie réformatrice du $\mathrm{VI}^{\mathrm{e}}$ siècle, la Vie des Pères du Jura, se fait l'écho de ces débats. Dans ce grand récit tripartite admirablement composé et doté du titre éloquent de Vita vel regula, l'auteur propose aux moines d'Agaune une série d'exempla très concrets afin de les exhorter à se conformer aux enseignements des trois pères fondateurs du Jura. Le mode de vie qui leur est proposé est celui d'un cénobitisme assez radical, qui tend à établir une séparation nette entre le monde des moines et celui du clergé. L'auteur anonyme de cette Vie estime en effet qu'un abbé ne doit pas être ordonné prêtre et que les clercs n'ont pas leur place au monastère. En filigrane, on peut lire dans cette Vie une critique implicite du monastère de Lérins, qui servait d'école de vie à de futurs évêques. Exclusivement adressée à des moines et de portée purement locale, cette œuvre n'a toutefois connu qu'une diffusion limitée ${ }^{10}$. De manière générale, le monachisme de cette période se caractérise donc par son extrême diversité - une situation qui conduira le pape Grégoire le Grand à promouvoir, à la fin du siècle, une réorganisation générale de la vie monastique en Italie ${ }^{11}$.

10. Sur la Vita patrum Jurensium (éd. citée n. 3), voir en dernier lieu A. DubreucQ, «Lérins et la Burgondie dans le haut Moyen Âge », dans Y. Codou et M. Lauwers éd., Lérins, une île sainte..., p. 195-227.

11. G. JENAL, «In cerca di ordine quando l'apocalisse sembra vicina: Gregorio Magno e il monachesimo del suo tempo in Italia», dans Gregorio Magno nel XIV centenario della sua morte. Convegno internazionale, Rome, 2004, p. 221-246. 


\section{La floraison monastique du VII ${ }^{\mathrm{e}}$ siècle et ses conséquences}

Dans le monde franc, le processus de réforme monastique connaît une nouvelle impulsion au début du $\mathrm{VII}^{\mathrm{e}}$ siècle, cette fois grâce à l'appui, voire à l'initiative, des rois et de leur entourage, à partir de Clotaire II et de son fils Dagobert ${ }^{\mathrm{I} r}$. Après la réunification des tria regna en 613 , les relations entre le roi et l'aristocratie - évêques compris - doivent être redéfinies. Dans le même temps, le monachisme connaît une promotion spectaculaire. Avec le soutien de toutes les grandes familles, les nouvelles fondations se multiplient surtout dans les régions septentrionales du royaume: sur les 320 nouveaux monastères recensés au $\mathrm{VII}^{\mathrm{e}}$ siècle, on en compte 230 au nord de la Loire et 90 au sud ${ }^{12}$. Comme le souligne Martin Heinzelmann, ces nouveaux monastères bousculent le pouvoir traditionnel de l'évêque dans son diocèse, d'un point de vue spirituel autant qu'économique ${ }^{13}$. Même si nombre d'évêques sont issus du monde monastique, même s'ils appartiennent souvent aux mêmes familles que celles des fondateurs de monastères, les tensions observées auparavant entre évêques et abbés, entre clercs et moines, s'exacerbent au cours du viI siècle, notamment sur la question des biens cédés aux églises.

Une nouvelle réforme s'impose: afin d'unifier le royaume, il convient de préserver les prérogatives traditionnelles de l'Église séculière sans pour autant léser les nouvelles fondations monastiques des aristocrates. Durant deux siècles, les rois successifs vont s'efforcer de promouvoir un processus réformateur dont les étapes sont encore mal connues, en premier lieu parce que l'importance de la réforme carolingienne, considérée comme l'acte de naissance du monachisme bénédictin, a eu tendance à éclipser les tentatives antérieures. Longtemps, l'historiographie traditionnelle a mis en exergue la figure du célèbre Colomban, qui aurait généré un «réseau monastique» qualifié d'iro-franc ou colombanien dont la spécificité aurait été la promotion d'une règle mixte inspirée à la fois de Benoît de Nursie et de Colomban. Au milieu du siècle, la reine Bathilde aurait apporté son appui à ce réseau en imposant cette même règle aux basiliques principales du royaume. À la fin du même siècle, la figure de Colomban se serait estompée au profit du seul Benoît, privilégié par la famille des Pippinides alors en pleine ascension. Lorsque Pépin III fut en mesure de promouvoir une réforme générale de l'Église franque, la règle bénédictine se serait progressivement répandue

12. P. Fouracre et R. Gerberding, Late Merovingian France. History and Hagiography 640720, Manchester-New York, 1996, p. 8.

13. M. Heinzelmann, «L'hagiographie mérovingienne. Panorama des documents potentiels», dans M. Goullet, M. Heinzelmann et C. Veyrard-Cosme éd., L'Hagiographie mérovingienne à travers ses réécritures, Ostfildern, 2010, p. 27-82 (p. 66). 
partout avant de voir son triomphe consacré sous Louis le Pieux aux conciles d'Aix de 816-817, qui voulurent l'imposer à tous les monastères de l'Empire ${ }^{14}$. De nombreuses recherches récentes ont démontré les faiblesses de cette reconstitution, sans pour autant résoudre tous les problèmes. L'importance accordée à Colomban découle en réalité de la Vie de Colomban et de ses disciples, très diffusée en Gaule franque ${ }^{15}$. Or, ce texte ne reflète que le point de vue de son auteur, Jonas de Suse, en 641-642: malgré ses efforts pour réhabiliter la figure de son maître mort en exil, le monachisme de cette période était beaucoup plus diversifié qu'il n'y paraît ${ }^{16}$. Compte tenu de la polysémie du terme de «règle», il n'est plus question de projeter sur les communautés mérovingiennes la conception moderne de l'observance littérale à un règlement écrit ${ }^{17}$. Plus que la diffusion de la règle bénédictine, le véritable enjeu des réformes de cette période réside dans la distinction nette qu'il s'agissait d'établir entre un «ordre des clercs » et un «ordre des moines » dont les fonctions devaient être définies de plus en plus précisément ${ }^{18}$.

14. Cette reconstitution remonte à la synthèse, aujourd'hui controversée, de F. Prinz, Frühes Mönchtum im Frankenreich. Kultur und Gesellschaft in Gallien, den Rheinlanden und Bayern am Beispiel der monastischen Entwicklung (4. bis 8. Jahrhundert), Munich-Vienne, 1965, rééd. Darmstadt 1988.

15. Vita Columbani et discipulorum eius auctore Iona (BHL 1898), éd. B. Krusch, Ionae vitae sanctorum Columbani, Vedastis, Iohannis, Hanovre-Leipzig, 1905 (MGH, Scriptores rerum germanicarum in usum scholarum, 37), p. 144-224. Sur sa date et son contexte, voir en dernier lieu I. Wood, «Jonas, the Merovingians, and Pope Honorius : Diplomata and the Vita Columbani », dans A. C. Murray éd., After Rome's Fall. Narrators and Sources of Early Medieval History. Essays presented to Walter Goffart, Toronto, 1998, p. 99-120.

16. Voir surtout A. DieRKENs, «Prolégomènes à une histoire des relations culturelles entre les îles britanniques et le continent pendant le haut Moyen Âge. La diffusion du monachisme dit colombanien ou iro-franc dans quelques monastères de la région parisienne au $\mathrm{VII}^{\mathrm{e}}$ siècle et la politique religieuse de la reine Bathilde», dans H. Atsma éd., La Neustrie. Les pays au Nord de la Loire de 650 à 850. Colloque historique international, Sigmaringen, 1989, t. 2, p. 371-394.

17. Voir A. A. HÄussLING, Mönchskonvent und Eucharistiefeier. Eine Studie über die Messe in der abendländischen Klosterliturgie des frühen Mittelalters und zur Geschichte der Messhäufigkeit, Münster, 1973; G. Moyse, «Monachisme et réglementation monastique en Gaule avant Benoît d'Aniane», dans J. DuBoIs éd., Sous la règle de saint Benoît. Structures monastiques et sociétés en France du Moyen Âge à l'époque moderne, Genève-Paris, 1982, p. 3-19; A. DIEM, «Was bedeutet "regula Columbani"? », dans W. PoHL et M. DieSENBERGER éd., Integration und Herrschaft. Ethnische Identitäten und soziale Organisation im Frühmittelalter, Vienne, 2002, p. 63-89.

18. Voir notamment J. SEmmler, «Benedictus II : una regula - una consuetudo», dans W. Lourdaux et D. Verhelst éd., Benedictine Culture 750-1050, Louvain, 1983, p. 1-49; ID., «Le monachisme»; M. DE JonG, «Carolingian monasticism: the power of prayer», dans R. MCKITTERICK éd., The New Cambridge Medieval History, II, c. 700-c. 900, Cambridge, 1995, p. 622-653; M. GAILlARD, D'une réforme à l'autre (816-934): les communautés religieuses en Lorraine à l'époque carolingienne, Paris, 2006, p. 123-147. 
La question des relations entre l'Église séculière et les monastères est donc centrale. Pour l'étudier, nous disposons de différents types de sources: outre les récits hagiographiques, il s'agit de quelques lettres et canons de conciles, d'une trentaine de textes qualifiés de «règles » ${ }^{19}$ et surtout d'une série d'actes diplomatiques, en particulier ceux qui octroient aux monastères des privilèges que Eugen Ewig a qualifiés de «grande» et de «petite liberté ${ }^{20}$. Concédés par des évêques ou des rois, ils visent à protéger les moines et leurs biens contre les ingérences de leur évêque diocésain, apportant ainsi quelques nuances au fameux concile de Chalcédoine de 451 qui plaçait les monastères sous le contrôle des évêques ${ }^{21}$. Certains de ces actes se réfèrent explicitement aux quatre communautés qui avaient été les premières, en Gaule, à bénéficier de ce statut privilégié et apparaissent à ce titre comme des modèles : Lérins, Saint-Maurice d'Agaune, Luxeuil et Saint-Marcel de Chalon-sur-Saône ${ }^{22}$. Restent enfin les sources hagiographiques qui, en raison des difficultés que pose leur examen critique, n'ont que rarement été étudiées dans cette perspective. Heureusement, leur corpus peut aujourd'hui être établi de manière plus précise grâce aux nombreuses recherches récentes qui leur ont été consacrées ${ }^{23}$.

\section{L'apport de l'hagiographie à l'étude des réformes mérovingiennes}

Après la réunification du royaume en 613 , les rois et leur entourage vont s'appuyer sur une partie des nouveaux monastères en les affectant au service du royaume. Afin d'unifier l'aristocratie des tria regna après les troubles générés par la guerre civile, les rois confient aux moines la tâche de «corriger» les grands du royaume en leur inculquant la pénitence et la crainte de Dieu. Comme je l'ai montré ailleurs - et l'on me permettra de résumer ici brièvement mes

19. A. DE VoGÜÉ, Les Règles monastiques anciennes (400-700), Turnhout, 1985.

20. La plupart des articles publiés par Eugen Ewig sur la question de 1968 à 1973 ont été réédités dans E. EwIG, Spätantikes und fränkisches Gallien. Gesammelte Schriften (1952-1973), H. ATsmA éd., 2 vol., Zurich-Munich, 1976-1979. On y ajoutera ID., «Bemerkungen zu zwei merowingischen Bischofsprivilegien und einem Papstprivileg des 7. Jahrhunderts für merowingische Klöster», dans A. Borst éd., Mönchtum, Episkopat und Adel zur Gründungszeit des Klosters Reichenau, Sigmaringen, 1974, p. 215-250.

21. Sur les canons de Chalcédoine relatifs aux moines, voir G. DAGRON, «Les moines et la ville. Le monachisme à Constantinople jusqu'au concile de Chalcédoine (451)», Travaux et mémoires, 4 (1970), p. 229-276.

22. Pour une synthèse récente, on préférera A. Dierkens, «Prolégomènes... », p. 388-393, à la thèse moins convaincante de B. H. RosenweIn, Negotiating Space. Power, Restraint, and Privileges of Immunity in Early Medieval Europe, Ithaca-Londres, 1999, p. 74-96.

23. Voir la magistrale synthèse des travaux récents établie par M. Heinzelmann, «L'hagiographie mérovingienne...». 
conclusions ${ }^{24}$-, les monastères privilégiés par les rois servent ainsi à la fois de prison et d'école, où les valeurs monastiques sont enseignées à des aristocrates qui n'ont pas forcément vocation à devenir moines. De fait, les vertus de perfection chrétienne n'offrent-elles pas la meilleure formation possible à tous ceux qui auront pour mission de participer au ministère royal? Une série de récits hagiographiques «réformateurs » vont alors s'efforcer de promouvoir leur propre système de valeurs, destiné à définir non seulement les normes qui doivent régir le «monachisme régulier», mais aussi les vertus que doivent acquérir les serviteurs de l'État.

La Vie de Colomban et de ses disciples offre ainsi un bel exemple de Vie réformatrice, qui vise à la fois à définir une forme bien précise de monachisme régulier et à donner à cet idéal un caractère universel en le proposant comme la base d'un système de gouvernement. Dans le premier livre, Jonas s'efforce de réhabiliter la figure de Colomban en insistant sur son héritage spirituel, censé se transmettre à toutes les personnes qui sont entrées en contact avec lui ou l'un de ses successeurs, ce qui inclut une bonne partie de l'aristocratie franque. Jonas y développe sa vision de ce qu'il nomme la «vertu de religion» : pour lui, l'humilité mutuelle des moines s'oppose à la compétition qui anime les hommes séculiers dans leur quête des honores. Aux vertus des premiers - piété, charité envers tous, modestie et sobriété, mansuétude et douceur - s'opposent les vices des seconds - paresse et discorde, orgueil et arrogance, colère et jalousie. Il exhorte ainsi les aristocrates à apprendre à renoncer à leurs propres volontés afin d'œuvrer en faveur de l'intérêt général, en s'inspirant de la vie commune des moines de Luxeuil ${ }^{25}$.

La suite de l'œuvre, composée des Vies d'Athala de Bobbio, d'Eustaise de Luxeuil, des moniales de Faremoutiers et de Bertulphe de Bobbio, répond à des intentions plus diversifiées qui correspondent aux attentes ponctuelles de chacune des communautés concernées ${ }^{26}$. Toutefois, le tableau d'ensemble qui se dégage de l'œuvre entière propose un nouveau modèle de « discipline régulière » qui, bien que présenté par Jonas comme «règle de Colomban», s'écarte assez nettement des enseignements de l'abbé irlandais ${ }^{27}$. Les grands principes en sont

24. A.-M. HelvéTIUS, «Hagiographie et formation politique des aristocrates dans le monde franc (VII ${ }^{\mathrm{e}}$-VIII ${ }^{\mathrm{e}}$ siècles)», dans E. BozoKy éd., Hagiographie, idéologie et pouvoir au Moyen Âge. Actes du colloque de Poitiers, septembre 2008, Turnhout (sous presse).

25. Vita Columbani, passim. On trouvera les références précises dans A.-M. HeLvétius, «Hagiographie...».

26. I. Wood, «Jonas...».

27. Sans revenir ici sur les controverses relatives aux règles qui nous sont parvenues sous le nom de Colomban, ses enseignements sont surtout connus par sa correspondance : Sancti Columbani Opera, G. S. M. WALKER éd., Dublin, 1957 (rééd. 1970), également accessible en ligne sur le site du Corpus of Electronic Texts: http://celt.ucc.ie. 
les suivants: les moines doivent vivre à l'intérieur d'un espace réservé, fermé aux visiteurs, mais dont eux-mêmes peuvent sortir selon les nécessités; ils ne sont pas astreints à la stabilité ; ils pratiquent le travail manuel; leurs offices ne sont pas accessibles aux séculiers; en échange d'une protection royale (Luxeuil) ou pontificale (Bobbio), ils échappent à l'autorité de leur évêque diocésain; ils peuvent assurer un service public tel que l'éducation des aristocrates, la garde des prisonniers politiques et l'accueil des pauvres et des voyageurs, mais dans des édifices séparés de leur propre clôture. Le roi peut également les envoyer en mission d'évangélisation. Contrairement aux moines, les moniales n'assument aucune fonction publique, mais sont astreintes à la stabilité, soumises à une clôture très stricte et placées sous la surveillance de moines expérimentés. En revanche, moines et moniales n'assurent pas la prière pour le roi ou le royaume ${ }^{28}$, ni le culte des reliques, ni les sépultures ou les prières pour les défunts ${ }^{29}$.

De manière plus générale, Jonas se montre convaincu de la supériorité des enseignements monastiques sur ceux de l'Église séculière, place les abbés sur un pied d'égalité avec les évêques et proclame son attachement au pape et sa vénération pour saint Pierre, prince des apôtres. Il fait preuve d'une extrême sévérité à l'encontre des hérétiques, des schismatiques et de tous ceux qui s'écartent de la voie droite, voués à la vengeance divine sans espoir de pardon. Ces particularités s'expliquent par le contexte: Jonas a été directement confronté aux problèmes posés par la royauté lombarde, de confession arienne, et par le schisme tricapitolin ${ }^{30}$; il a en outre eu l'occasion de rencontrer personnellement le pape Honorius, qui a pris le monastère de Bobbio sous sa protection contre l'évêque de Tortone ${ }^{31}$. Pour lui, l'autorité du pape prime donc sur celle du concile, y compris celui de Chalcédoine.

Le modèle de monachisme proposé par Jonas en 641-642 était en contradiction avec les traditions monastiques anciennes de la Gaule. De toute évidence, le silence prudent qu'il observe sur le règne de Dagobert $\mathrm{I}^{\mathrm{er}} \mathrm{s}$ 'explique par le fait que ce roi avait accordé son soutien à d'autres types de communautés, et en

28. Vita Columbani, I, 28: Colomban refuse de prier pour le roi au nom de Mt 5, 44: «aimez vos ennemis et priez pour ceux qui vous persécutent ». Athala applique ce même commandement au livre II, 24. La Vie diverge ici par rapport à la Regula coenobialis attribuée à Colomban (cf. I. Wood, «onas...», p. 107).

29. Toute la partie relative aux moniales de Faremoutiers vise à les convaincre de l'inutilité de la prière pour les défunts, dès lors que chacun doit racheter ses péchés par des pénitences de son vivant.

30. Sur ce point, voir en dernier lieu B. DumÉzIL, «L'affaire Agrestius de Luxeuil: hérésie et régionalisme dans la Burgondie du VII ${ }^{\mathrm{e}}$ siècle», Médiévales, 52 (2007), p. 135-152; et plus généralement C. SotineL, «L'échec en Occident: l'affaire des Trois Chapitres», dans J.-M. MAYEuR et al. éd., Histoire du christianisme des origines à nos jours, t. 3, Paris, 1998, p. 427-455.

31. Voir en dernier lieu I. Wood, «Jonas...», p. 113-120. 
particulier à celle de Saint-Denis, où il avait confié aux moines le culte public de la laus perennis telle qu'elle se pratiquait à Saint-Maurice d'Agaune ${ }^{32}$. À la même époque, la louange perpétuelle était aussi chantée dans le monastère double de Remiremont, alors en rivalité avec Luxeuil et Faremoutiers ${ }^{33}$. Aux yeux de Jonas, de telles communautés monastiques ouvertes au public ne pouvaient pas être considérées comme «régulières», car les saints moines et moniales y vivaient au contact des séculiers ${ }^{34}$.

Lorsque Jonas reprend la plume, en 659, pour rédiger son autre récit hagiographique, la Vie de Jean, abbé et fondateur du monastère de La Réôme (Moutiers-Saint-Jean) ${ }^{35}$, les temps ont changé. Depuis la mort de Clovis II, la reine Bathilde gouverne le royaume avec son fils Clotaire III et Jonas, devenu abbé, accomplit pour elle des missions diplomatiques. La Vie de Jean écrite dans ce contexte peut également être considérée comme une œuvre réformatrice, au sens où Jonas y recommande à nouveau à tous les chrétiens de se conformer au modèle monastique régulier, qualifié ici encore de «religion ${ }^{36}$. Le texte est avant tout destiné à convaincre les moines du monastère Saint-Jean, alors dirigés par l'abbé luxovien Hunna, que le mode de vie régulier était déjà celui de leur saint fondateur. Comme l'a souligné récemment Albrecht Diem, Jonas présente ici son modèle non plus comme une nouveauté irlandaise, mais au contraire comme l'héritage de la plus célèbre tradition monastique gauloise, celle de Lérins au

32. Cf. acte de Clovis II de 654, éd. T. KöLzer, Die Urkunden der Merowinger, MGH, DDMerov., I, Hanovre, 2001, p. 219 ( $\mathrm{n}^{\circ}$ 85) et FRÉDÉGAIRE, Chronique des temps mérovingiens (Livre IV et continuations), éd. et trad. O. Devillers et J. Meyers, Turnhout, 2001, ch. 79. Voir P. BERNARD, «La laus perennis dans la Gaule de l'Antiquité tardive: état des questions et éléments d'un bilan», dans F. Bernabei et A. Lovato éd., Sine musica nulla disciplina... Studi in onore di Giulio Cattin, Padoue, 2006, p. 50-52; à compléter par J. BARBIER, « Le testament d'Ermentrude, un acte de la fin du vi ${ }^{\mathrm{e}}$ siècle?», Bulletin de la Société des Antiquaires de France, 2003 (2009), p. 135138.

33. Voir en dernier lieu A.-M. Helvétius et M. Gaillard, «Production de textes et réforme d'un monastère double : l'exemple de Remiremont du vII au IX siècle», dans J. F. HAMBURGER, C. JÄGGI, S. Marti et H. RöcKelein éd., Frauen - Kloster - Kunst. Neue Forschungen zur Kulturgeschichte des Mittelalters, Turnhout, 2007, p. 383-393.

34. On trouve déjà l'opposition entre in secreto et in publico - mais dans un autre contexte chez Colomban, Epistolae, 5.

35. Vita Iohannis abbatis Reomaensis auctore Iona (BHL 4424), éd. B. Krusch, Ionae vitae, p. 321-344. À mon sens, la troisième Vie attribuée à Jonas, celle de Vaast d'Arras, n'est pas de lui : cf. A.-M. Helvétrus, «Clercs ou moines? Les origines de Saint-Vaast d'Arras et la Vita Vedastis attribuée à Jonas », Revue du Nord (Mélanges offerts à Stéphane Lebecq), sous presse.

36. Sur le sens du terme, voir H. Atsma, «Die christlichen Inschriften Galliens als Quellen für Klöster und Klosterbewohner bis zum Ende des 6. Jahrhunderts », Francia, 4 (1976), p. 40-42 et 44-46 (avec bibliographie). 
temps de son fondateur Honorat et celle des Conférences de Jean Cassien ${ }^{37}$. Si l'idéal de vie régulière proposé dans la Vie de Jean présente certes des similitudes avec celui que Jonas attribuait à Colomban dans son œuvre antérieure, il existe aussi des différences qui méritent de retenir l'attention ${ }^{38}$.

D'abord, parmi les vertus que prêchait l'abbé Jean «tant aux moines qu'au peuple», nous trouvons en premier lieu l'humilité et l'obéissance, mais ces dernières font l'objet d'un nouveau développement. Plutôt que d'obéir au seul maître, il s'agit cette fois d'obéir à tous. Contrairement à la Vie de Colomban qui encensait l'autorité des abbés, la Vie de Jean nous offre le portrait d'un père plus humble, qui préfère «se soumettre à tous en obéissant que diriger en dominant ${ }^{39} »$. Par conséquent, il n'hésite pas à abandonner son troupeau pour se retirer à Lérins afin d'y apprendre «la discipline des anciens pères sous la rigueur de l'obéissance ${ }^{40} \gg$. Surtout, cette obéissance de l'abbé s'applique désormais aussi aux évêques - non seulement à l'évêque diocésain Grégoire de Langres qui ordonne à Jean de réintégrer son monastère, mais aux évêques en général lorsqu'il s'agit d'ériger un autel. En outre, si les moines sont toujours encouragés à vivre dans leur clôture en refusant les visites des laïcs, Jonas semble tolérer une solution de compromis en matière d'accueil des fidèles et de promotion du culte de Jean. Ainsi, les moines sont invités à distribuer, aux portes du monastère, des eulogies ou de l'eau de la fontaine sacrée aux laïcs qui le demandent; quant au corps de Jean, à en croire Jonas, il aurait été transféré «sur le conseil des évêques » dans un nouveau sanctuaire public situé en dehors de la clôture, où sont célébrés aussi bien les messes que les offices de prières et où sont accueillis les malades ${ }^{41}$.

Dans la Vie de Jean, Jonas cherche à convaincre les moines et les grands que le monastère de La Réôme était déjà régulier, au sens où il l'entend, lors de

37. A. DiEm, «The rule of an "iro-egyptian" monk in Gaul : Jonas'Vita Iohannis and the construction of a monastic identity», Revue Mabillon, 80 (2008), p. 5-50. Sur la règle de Macaire, présentée par Jonas comme le fondement de l'idéal monastique de Jean, je n'adhère pas aux hypothèses d'A. Diem. Compte tenu du contexte langrois, il est plus vraisemblable d'identifier ce Macaire avec le Macaire d'Antioche exilé en Cappadoce qui est présenté comme le père spirituel des Trijumeaux de Langres dans leur Passion: Passio Speusippi, Eleusippi, Meleusippi tergemini (BHL 7828), ch. 7, éd. AASS., Jan. II, p. 75. En effet, ce texte fut vraisemblablement rédigé durant l'épiscopat de Grégoire de Langres (506/507-539/540), auquel notre Jean de Réôme est associé (cf. M. Heinzelmann, «L'hagiographie mérovingienne...», p. 42 et n. 63).

38. Contrairement à ce qu'affirme A. DIEM («The rule...», p. 50), le modèle de la Vie de Jean ne peut donc pas être considéré seulement comme une nouvelle Regula Columbani. Le nom de Colomban n'est d'ailleurs pas mentionné dans la Vie de Jean.

39. Vita Johannis, 3 et 4.

40. Jonas parle ici de la Lérins de l'ancien temps, celle qui était restée fidèle aux enseignements de son fondateur Honorat; dans la Vie de Colomban, en effet, Jonas ne considérait plus Lérins comme une communauté « régulière » (Vita Columbani, II, 1).

41. Vita Iohannis, 20. 
sa fondation - ce qui, assurément, ne correspondait pas à la réalité historique : aux origines, il s'agissait d'une communauté ouverte, dotée d'un sanctuaire à reliques et d'une fontaine sacrée, plus proche sans doute du modèle de Saint-Maurice d'Agaune que de celui de Lérins ${ }^{42}$. Vers la fin du $\mathrm{vl}^{\mathrm{e}}$ siècle, les lieux avaient été réaménagés en vue de séparer l'espace monastique des parties accessibles au public $^{43}$. En 659, l'abbé Hunna souhaitait parachever cette réforme régulière afin, peut-être, de pouvoir bénéficier d'un privilège de protection royale ${ }^{44}$. Mais à l'époque de la reine Bathilde, de tels privilèges n'accordaient plus que la «petite liberté» et non la «grande» dont avaient pu bénéficier Luxeuil et quelques autres communautés. Cette liberté plus restreinte résultait d'un compromis établi par Clovis II et Bathilde en vue de préserver la juridiction des évêques diocésains sur les monastères, conformément au concile de Chalcédoine ${ }^{45}$. En réécrivant l'histoire du monastère de La Réôme dans sa Vie de Jean, Jonas tente de concilier les intérêts de l'abbé Hunna et ceux de la reine. Il aménage son idéal monastique régulier dans le sens d'une via media susceptible de ménager l'esprit d'indépendance des moines et l'autorité des évêques. Mais il cherchait ainsi à concilier l'inconciliable, et la diffusion très limitée de sa Vie de Jean témoigne de l'échec de l'entreprise. Le modèle monastique ici proposé, nettement moins homogène que celui de la Vie de Colomban, ne pouvait guère susciter l'enthousiasme dans le royaume.

Les modalités précises de la réforme monastique promue par la reine Bathilde continuent de faire l'objet de débats entre les historiens. Le sanctus regularis ordo qu'elle impose aux basiliques majeures ${ }^{46}$ est aussi celui qu'elle promeut à Chelles et à Corbie ${ }^{47}$ : à mon sens, l'expression ne désigne ni une organisation de vie commune spécifique, ni un statut juridique particulier, mais plutôt l'adhésion à un état d'esprit, à un ensemble de valeurs, à une conception globale de la «discipline régulière» telle qu'on pouvait se la représenter par

42. La référence à la «règle de Macaire» et au rôle de Grégoire de Langres (cf. supra, n. 37) témoigne du fait que Jonas devait disposer d'écrits aujourd'hui perdus. Le texte qui nous a été transmis sous le nom de Regula Macarii a subi de nombreuses interpolations dont l'éditeur minimise à mon avis la portée (éd. A. DE VogüÉ, Les Règles des saints pères, I, Paris, 1982, p. 372-389).

43. Vita Iohannis, 20: il s'agit du transfert du tombeau de Jean dans une nouvelle basilique publique. Jonas situe l'événement sous l'épiscopat de Mummulinus de Langres, devenu évêque vers 579-580. La translation eut lieu le jour de la saint Maurice, preuve de l'existence d'un lien spirituel avec Agaune.

44. A. Diem, «The rule...», p. 10, n. 23.

45. Cf. supra, n. 20-22.

46. Selon la formule utilisée peu après 680 dans Vita Balthildis prima (BHL 905), 9, éd. B. KRUSCH, MGH, SSRM, II, Hanovre, 1889, p. 493.

47. Voir L. Morelle, «Le statut d'un grand monastère franc: Corbie (664-1050)», dans F. Bougard éd., Le Christianisme en Occident du début du vII a au milieu du XI siècle. Textes et documents, Paris, 1997, p. 205-215. 
référence à Benoît, à Colomban et aux autres pères. Comme le souligne son hagiographe, Bathilde elle-même se comporte ut vera monacha après sa retraite à Chelles ${ }^{48}$. Les communautés ainsi reconnues comme régulières méritent d'obtenir un privilège de liberté à la condition que leurs moines acceptent de se soumettre à l'autorité d'un supérieur et de renoncer à leurs volontés propres afin d'œuvrer en faveur de l'intérêt général du royaume. En l'occurrence, les moines y ont le devoir de prier «pour le maintien de l'Église et le salut des rois, pour la stabilité du royaume et la tranquillité de la patrie ${ }^{49} »$, l'efficacité de leur prière étant garantie par le respect de la discipline régulière.

Les efforts de Bathilde seront toutefois ruinés par les conflits politiques qui affectèrent le royaume à la fin du viI ${ }^{\mathrm{e}}$ et au début du VIII ${ }^{\mathrm{e}}$ siècle. Durant cette période furent rédigés de nombreux textes hagiographiques, dont quelquesuns répondent à des intentions réformatrices qui apparaissent comme de plus en plus diversifiées. C'est le cas, par exemple, de la Vie tripartite des abbés de Remiremont Amé, Romaric et Adelphe, qui, comme nous l'avons montré ailleurs, fut écrite vers 675-680 à la fois dans une perspective de conciliation politique et de promotion d'une réforme monastique ayant pour but de séparer les moniales débutantes, astreintes au travail manuel dans un cénobe de type bénédictin, et les moniales aguerries, affectées au chant de la laus perennis sur le saint Mont ${ }^{50}$. D'autres auteurs s'efforcent toujours d'inculquer les valeurs monastiques aux laïcs, comme dans la Vie de saint Wandrille de Fontenelle vers 700, qui nous montre des «hommes brutaux et cruels», une fois convertis par la prédication du saint homme, se prosterner jusqu'à terre «à la façon des moines » et demander pardon ${ }^{51}$. Les évêques aussi sont encouragés à s'inspirer des exemples des moines, comme dans la très polémique Vie de Philibert de Jumièges vers $750^{52}$, où l'on voit l'évêque Ansoald de Poitiers prêt à s'en remettre aux conseils de l'abbé Philibert et à «incliner sa puissance épiscopale sous la norme de la religion ${ }^{53}$ ». À en croire l'hagiographe, la «religion» de Philibert formait une synthèse de nombreuses règles, car l'abbé avait su puiser à toutes les

48. Vita Balthildis, 19, p. 507. Sur cette expression, déjà présente au $\mathrm{VI}^{\mathrm{e}}$ siècle dans la Vie d'Antoine de Lérins, cf. S. GiOANNI, «Être véritablement moine...».

49. C'est le cas pour Corbie, soumis à la «Règle de Benoît et Colomban», aussi bien que pour Saint-Denis, qui pratique la laus perennis d'Agaune. Cf. l'acte de Berthefrid d'Amiens pour Corbie en 664, trad. L. Morelle, «Le statut...», p. 207, et l'acte de Clovis II pour Saint-Denis en 654 (éd. citée n. 32).

50. A.-M. Helvétius et M. Gaillard, «Production de textes... ».

51. Vita Wandregisili Fontanellensis (BHL 8804), 16, éd. B. Krusch, MGH, SSRM, V, HanovreLeipzig, 1910, p. 21. Voir aussi A.-M. Helvétıus, «Hagiographie... ».

52. Vita Filiberti Gemeticensis (BHL 6805), éd. W. Levison, MGH, SSRM, V, Hanovre-Leipzig, 1910, p. 583-604.

53. Vita Filiberti, 26. 
sources, «comme une abeille prudente ${ }^{54} »$ : «Il fréquentait par une lecture assidue les charismata de saint Basile, la règle de Macaire, les décrets de Benoît, les très saintes institutions de Colomban; c'est ainsi, chargé par la vertu des aromates, qu'il montrait son saint exemple à ceux qui le suivaient ${ }^{55}$.»

$\mathrm{Au}$ terme de cette présentation, j'espère avoir convaincu le lecteur de l'intérêt que peut présenter l'analyse des Vies de saints, dès lors qu'on les confronte aux autres sources, pour l'étude des réformes monastiques précarolingiennes. Il est bien connu que les monastères étaient des centres de pouvoir politique essentiels dans la société franque ${ }^{56}$. Enjeux de rivalités et objets de convoitise entre les factions de l'aristocratie, ils étaient aussi des lieux d'écriture capables de produire des textes de propagande susceptibles de servir les intérêts de leurs protecteurs. L'un des supports privilégiés de cette propagande - non le seul -était la Vie du saint fondateur, qui se prêtait à merveille à la réécriture du passé sous le couvert de la fidélité à la tradition des origines. L'exemple du premier père, pasteur et forma de son troupeau, devait servir à réformer, à corriger les mœurs de la communauté, voire de toute la société. La diversité des modèles présentés reflète celle des intérêts variés que ces textes servaient à défendre, y compris sous la plume d'un même auteur, comme en témoigne le cas de Jonas de Suse.

Les différents courants réformateurs qui se manifestent durant cette période aboutiront, au terme d'un long processus, à l'unification des monastères sous la règle de saint Benoît. Or, comme l'a souligné Josef Semmler, le fait de vouloir imposer une règle unique aux monastères était contraire à toute la tradition monastique ${ }^{57}$. La portée de cette mesure s'éclaire à la lumière des enjeux politiques que représentaient les valeurs monastiques, dès lors que d'aucuns cherchaient à leur conférer un caractère universel et à les imposer comme modèle de gouvernement. L'histoire des courants de réformes du haut Moyen Âge reste mal connue, notamment en raison de l'état lacunaire de la documentation conservée. Il me semble néanmoins indispensable d'intégrer au corpus les récits hagiographiques, certes souvent décrits comme stéréotypés, mais dont certains recèlent des richesses insoupçonnées si l'on prend la peine de prêter attention à des éléments souvent négligés: les catalogues de vertus des saints, l'expression des doxologies, les références explicites ou implicites aux anciens pères méritent d'être étudiés de manière approfondie. Le phénomène des réformes monastiques sera mieux compris à la lumière de ce que dévoilent ces textes, au moment de

54. L'auteur s'inspire ici de la Vie d'Antoine, 3, 4.

55. Vita Filiberti, 5 .

56. Voir par exemple R. LE JAN, «Monastères de femmes, violence et compétition pour le pouvoir dans la Francie du vII ${ }^{\mathrm{e}}$ siècle», dans EAD., Femmes, pouvoirs et société dans le haut Moyen Âge, Paris, 2001, p. 89-107.

57. J. SemmLER, «Le monachisme occidental...», p. 82-83. 
leur rédaction, sur les relations entre moines et évêques, sur l'autorité de l'abbé, sur la présence ou non d'un sanctuaire ouvert au public, mais aussi sur les modalités précises de l'obéissance, de l'humilité, de la pénitence et des autres vertus recommandées par les différents auteurs à leur public.

Anne-Marie HelvéTıus - Université Paris 8, EA 1571, Centre de recherches historiques: Histoire des pouvoirs, savoirs et sociétés, 15-17 rue de CampoFormio, F-75013 Paris

\section{Hagiographie et réformes monastiques dans le monde franc du viI siècle}

Tandis que cohabitent diverses formes de monachisme dans le monde franc du $\mathrm{VII}^{\mathrm{e}}$ siècle, le rôle politique des monastères ne cesse de croître grâce au soutien des rois et de l'aristocratie. Une série de récits hagiographiques témoignent du lien étroit qui s'établit entre réforme monastique et unification politique. Dans cette perspective, la réforme acquiert parfois une dimension universelle: au-delà de la vie commune des moines, ce sont les valeurs chrétiennes et donc le comportement de tous les chrétiens que les hagiographes cherchent à corriger. La diversité des modèles présentés dans ces sources reflète celle des intérêts variés que ces textes servaient à défendre, y compris sous la plume d'un même auteur, comme en témoigne le cas de Jonas de Suse.

Haut Moyen Âge - royaume des Francs - hagiographie - monachisme aristocratie.

\section{Hagiography and Monastic Reforms in the VIIth Century Frankish World}

During the 7th century, the Frankish world hosted various forms of monasticism; meanwhile, as kings and the aristocracy supported the monasteries, their political role continuously grew up. A range of hagiographic narrative stresses out the close link between monastic reform and political unification. In this prospect, monastic reform sometimes aimed a larger audience: beyond the monastic way of life, the hagiographers define a system of Christian virtues that would be able to correct the behaviour of every faithful. In this matter, hagiographical sources offer a wide diversity of conceptions as they supported various peculiar interests; this seems obvious when we read an author like Jonas of Susa.

Early Middle Ages - Frankish kingdom - hagiography - monasticism aristocracy. 
